\title{
A Novel Event-Based Active Pixel Sensor for Cryo-EM Electron Counting
}

\author{
Benjamin Bammes ${ }^{1}$ and Robert Bilhorn ${ }^{2}$
}

${ }^{1}$ Direct Electron LP, Pearland, Texas, United States, ${ }^{2}$ Direct Electron LP, San Diego, California, United States

The introduction and widespread adoption of electron counting with direct detectors, along with improvements in software for image processing and 3D reconstruction, have propelled a "resolution revolution" in cryo-EM [1], so that atomic models of biological macromolecules are now routinely generated by the technique [2]. As a result, cryo-EM has become an essential tool in both basic biological research as well as drug discovery [3], with demand for cryo-EM equipment often exceeding availability [4].

One of the most significant bottlenecks for cryo-EM is the restrictive imaging conditions imposed by electron counting. Currently, electron counting is performed by acquiring a large number of frames from a high-sensitivity direct detector, with the electron beam sufficiently dim to yield sparse images of single electron events on each frame [5-6]. The sparsity within each frame is necessary to avoid coincidence loss stemming from the inability to discriminate multiple coincident electrons as separate events [5,7]. Operating outside of the sparse conditions for the camera has detrimental consequences, particularly for quantitative, low-dose applications such as cryo-EM. Therefore, at present, cryo-EM experiments are largely dictated by the specific exposure rate requirements of the electron counting camera.

Rethinking the strategy for electron counting, we have developed and implemented a novel large format $(4096 \times 4096)$ event-based monolithic active pixel sensor (MAPS) direct detection sensor (Fig. 1). Noise is minimized through on-chip correlated double sampling (CDS) and on-chip thresholding. Incident electrons are detected through an optimized sense amplifier, which encodes each detection event and sends it through high-speed digital input/output channels to on-board field programmable gate arrays (FPGAs), which perform sub-pixel precision centroiding on each event and accumulate super-resolution $(8192 \times$ 8192) dose-fractionated frames to be sent to the computer. To maximize resolution, this new sensor has a larger pixel size $(8 \mu \mathrm{m})$ than our previous direct detection sensors so that incident TEM primary electrons have a higher probability of being constrained a small number of pixels.

This novel sensor can detect and process incident electrons at an order of magnitude higher rate than brute force software-based counting detectors. The new detector maintains $>95 \%$ linearity up to 60 electrons per pixel per second at 200 and $300 \mathrm{kV}$, with high detective quantum efficiency ( >90\%) over at least three orders of magnitude of exposure rates (Fig. 2).

The camera has been successfully integrated in SerialEM for automated acquisition of both single particle and tomographic cryo-EM data.

This new strategy for electron counting promises to significantly improve the productivity and throughput of cryo-EM, by enabling high-speed acquisition of high-SNR datasets. Additionally, the simplicity of onchip electron counting reduces the overall cost of this camera, making high-resolution cryo-EM equipment more accessible. 


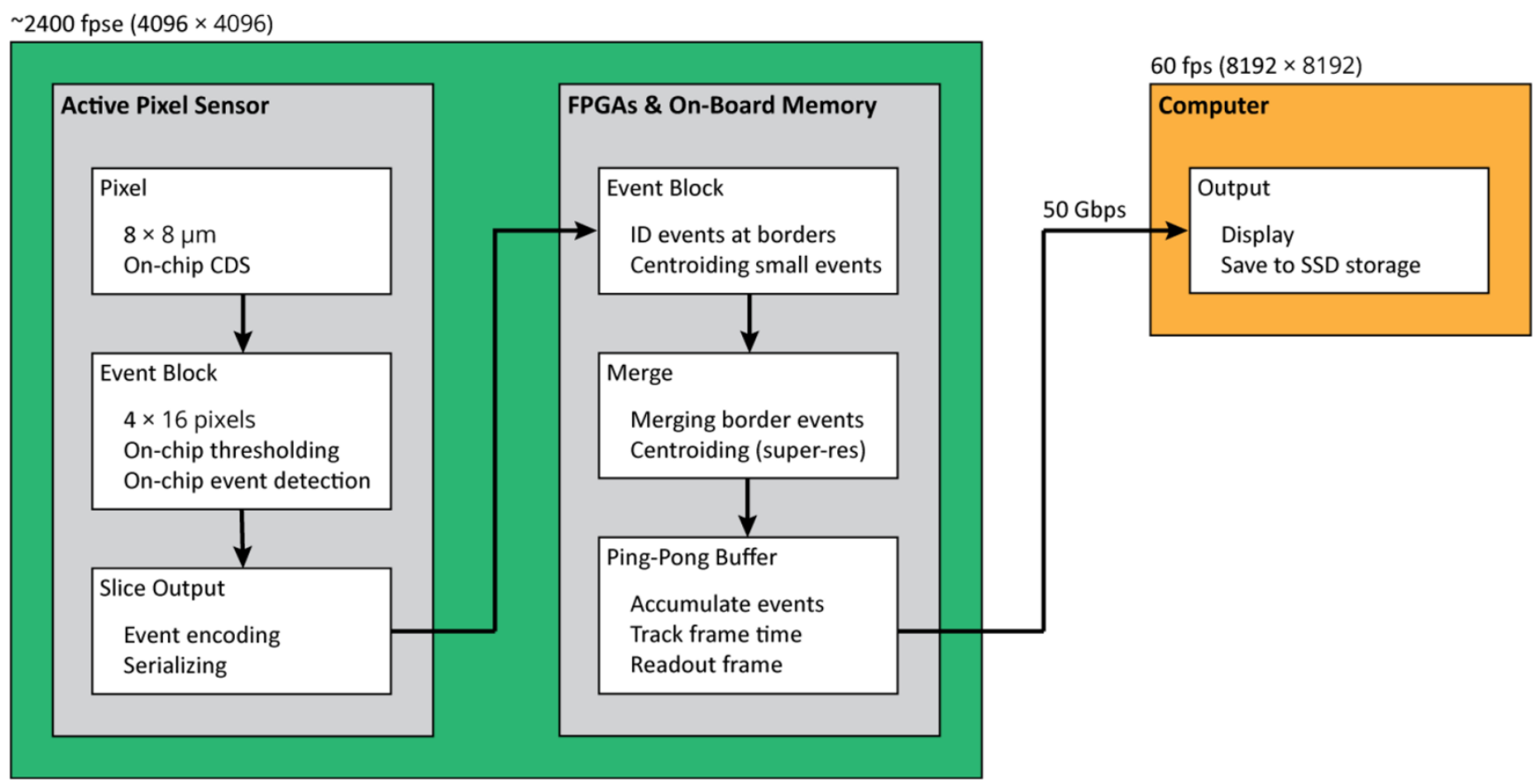

Figure 1. The overall architecture of the new camera. The green box denotes operations carried out within the electronics in the camera head.
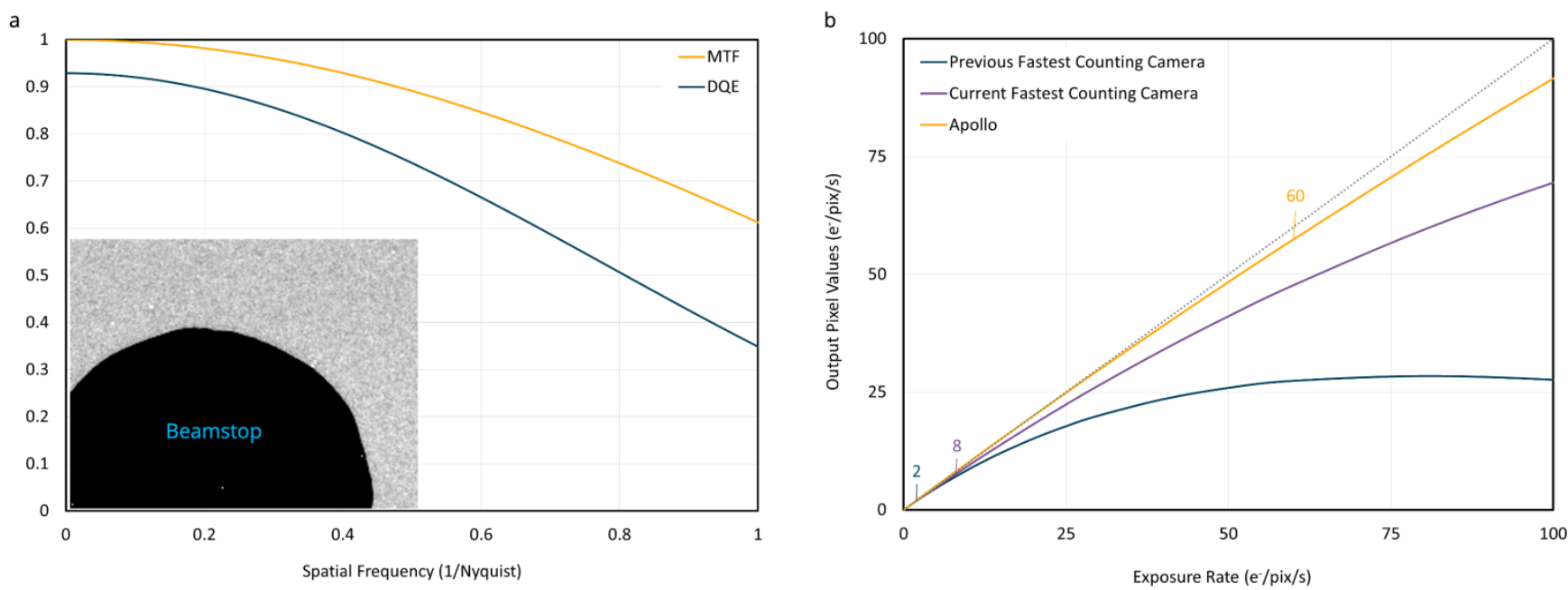

Figure 2. The performance of the new camera at $200 \mathrm{kV}$. (a) The modulation transfer function (MTF) and detective quantum efficiency (DQE). (b) The linearity of the camera at a variety of exposure rates compared to the linearity of fast brute-force electron counting cameras [7].

\section{References}

[1] Kühlbrandt W. (2014). The Resolution Revolution. Science, 343: 1443-1444.

[2] Nogales, E. (2016). "The development of cryo-EM into a mainstream structural biology technique." Nat Methods 13(1): 24-7.

[3] Renaud, J.P., Chari, A., Ciferri, C., Liu, W.T., Rémigy, H.W., Stark, H., \& Wiesmann, C. (2018). "Cryo-EM in drug discovery: achievements, limitations and prospects." Nat Rev Drug Discov 17(7): 47192. 
[4] Vinothkumar, K.R. \& Henderson, R. (2016). "Single particle electron cryomicroscopy: trends, issues and future perspective." Q Rev Biophys 49: e13.

[5] Jin L. (2009). Direct electron detection in transmission electron microscopy. University of California, San Diego.

[6] McMullan G., Clark A.T., Turchetta R., \& Faruqi A.R. (2009). Enhanced imaging in low dose electron microscopy using electron counting. Ultramicroscopy, 109: 1411-1416.

[7] Li X., Zheng S.Q. Egami K., Agard D.A., \& Cheng Y. (2013). Influence of electron dose rate on electron counting images recorded with the K2 camera. Journal of Structural Biology, 184: 251-260.

[8] This material is based upon work supported by the U.S. Department of Energy, Office of Science, under Award Number DE-SC0020577. 